\title{
PITCAIRNIA YOCUPITZIAE (BROMELIACEAE; PITCAIRNIOIDEAE), NOTABLE NUEVA ESPECIE DE GUERRERO, MÉXICO
}

\author{
Adolfo Espejo-Serna y Ana Rosa López-Ferrari
}

Universidad Autónoma Metropolitana - lztapalapa, División de Ciencias Biológicas y de la Salud, Departamento de Biología, Herbario Metropolitano, Apdo. postal 55535, 09340, México, D.F., México. aes@xanum.unam.mx

\section{RESUMEN}

Se describe e ilustra Pitcairnia yocupitziae Espejo \& López-Ferrarri, sp. nov. (Bromeliaceae), conocida únicamente del estado de Guerrero, México. La nueva especie se compara con P. roseana L. B. Sm., de la cual difiere por su inflorescencia y por sus brácteas florales. Se incluye también una clave para identificar las especies del género presentes en el estado de Guerrero.

Palabras clave: Bromeliaceae, Guerrero, Lista Roja IUCN, México, Pitcairnia.

\section{ABSTRACT}

Pitcairnia yocupitziae Espejo \& López-Ferrarri, sp. nov. (Bromeliaceae), known only from the state of Guerrero, Mexico, is described and illustrated. The new species is compared with $P$. roseana L. B. Sm., from which it differs by its inflorescence and floral bracts. An identification key for the species of Pitcairnia present in Guerrero is also included.

Key words: Bromeliaceae, Guerrero, IUCN Red List, Mexico, Pitcairnia.

Como parte de la revisión de material de herbario del género Pitcairnia con el propósito de elaborar la monografía de la familia Bromeliaceae para el país y como parte del proyecto específico Estudios Sistemáticos en el Género Pitcairnia (Bromeliaceae; Pitcairnioideae), encontramos material de un taxon no descrito, al que decidimos nombrar como: 
Pitcairnia yocupitziae Espejo \& López-Ferrarri, sp. nov. Fig. 1. B y D

Herba saxicola, caespitosa, florens ad $60 \mathrm{~cm}$ alta; folia basalia, dimorpha, viridia laminis linea recta transversa deciduis, altera castanea, persistentia. Inflorescentia terminalis, erecta, simplex, racemosa, fusiformis vel ellipsoidea, conspicue albido-lepidota; bracteis florigeris erectis, longe-ovatis, albido-lepidotis, acuminatis, sepala aequantibus vel superantibus; flores zygomorphi, breviter pedicellati; sepala viridia, lanceolata vel triangularia, conspicue albido-lepidota, adaxialia carinata; petala rubra, nuda, spathulata; stamina subaequalia, in corolla inclusa vel leviter exserta; stylo filiforme, exserto; ovario fere omnino supero, ovoideo; fructus ovoideus, semina rubra, bicaudata.

Plantas saxícolas, perennes, acaules, cespitosas, con rizomas subterráneos, formando grandes colonias, las rosetas bulbosas en la base, en flor de 60 a $75 \mathrm{~cm}$ de alto; raíces fibrosas, delgadas; hojas basales, dimorfas, unas reducidas, no fotosintetizadoras, con las vainas triangulares, de ca. $5 \mathrm{~cm}$ de largo, por ca. $1.8 \mathrm{~cm}$ de ancho, glabras en la parte adaxial, densamente blanco-lepidotas en la parte abaxial, verdes, pardas oscuras hacia la base en ambas superficies, enteras y con las láminas pardas, lineares a linear-triangulares, de 1.5 a $5.5 \mathrm{~cm}$ de largo, de 1 a $6 \mathrm{~mm}$ de ancho con el margen espinoso-serrado, persistentes; otras foliáceas, fotosintetizadoras, con las vainas orbiculares a muy ampliamente ovadas, de 2.4 a $4 \mathrm{~cm}$ de largo, de ca. $3 \mathrm{~cm}$ de ancho, pardas, y con las láminas verdes, lineares, de 40 a $75 \mathrm{~cm}$ de largo, de 1 a $1.5 \mathrm{~cm}$ de ancho, densa y conspicuamente blanco-lepidotas en el envés, glabras en la haz, enteras en el margen, largamente acuminadas y pungentes en el ápice, deciduas a lo largo de una línea transversal basal; inflorescencia terminal, simple, racemosa, polística, produciéndose en individuos que han perdido las hojas fotosintetizadoras; pedúnculo erecto a levemente curvado, cilíndrico, de 25 a $50 \mathrm{~cm}$ de largo, de 5 a $6 \mathrm{~mm}$ de diámetro, verde, conspicuamente blanco-lepidoto; brácteas del pedúnculo verdes, a veces rojizas en la base, erectas, lanceoladas, de 6 a 10 $\mathrm{cm}$ de largo, 1.5 a $2 \mathrm{~cm}$ de ancho, enteras, largamente acuminadas y pungentes en el ápice, conspicuamente blanco-lepidotas, reduciendo su tamaño paulatinamente hacia la porción apical del pedúnculo; racimo rollizo, elipsoide a fusiforme, de 17 a $20 \mathrm{~cm}$ de largo, de 4 a $5 \mathrm{~cm}$ de diámetro; brácteas florales rojas con el ápice pardo, erectas, largamente ovadas, de 4.5 a $5.5 \mathrm{~cm}$ de largo, 1.5 a $1.7 \mathrm{~cm}$ de ancho, casi tan largas o más largas que los sépalos, densa y conspicuamente blanco-lepidotas, acuminadas en el ápice; flores 20 a 25 por inflorescencia, cortamente pediceladas, zigomorfas, los pedicelos de ca. $4 \mathrm{~mm}$ de largo, rectos, conspicuamente blanco 
Espejo-Serna y López-Ferrari: Pitcairnia yocupitziae, nueva especie de Guerrero, México
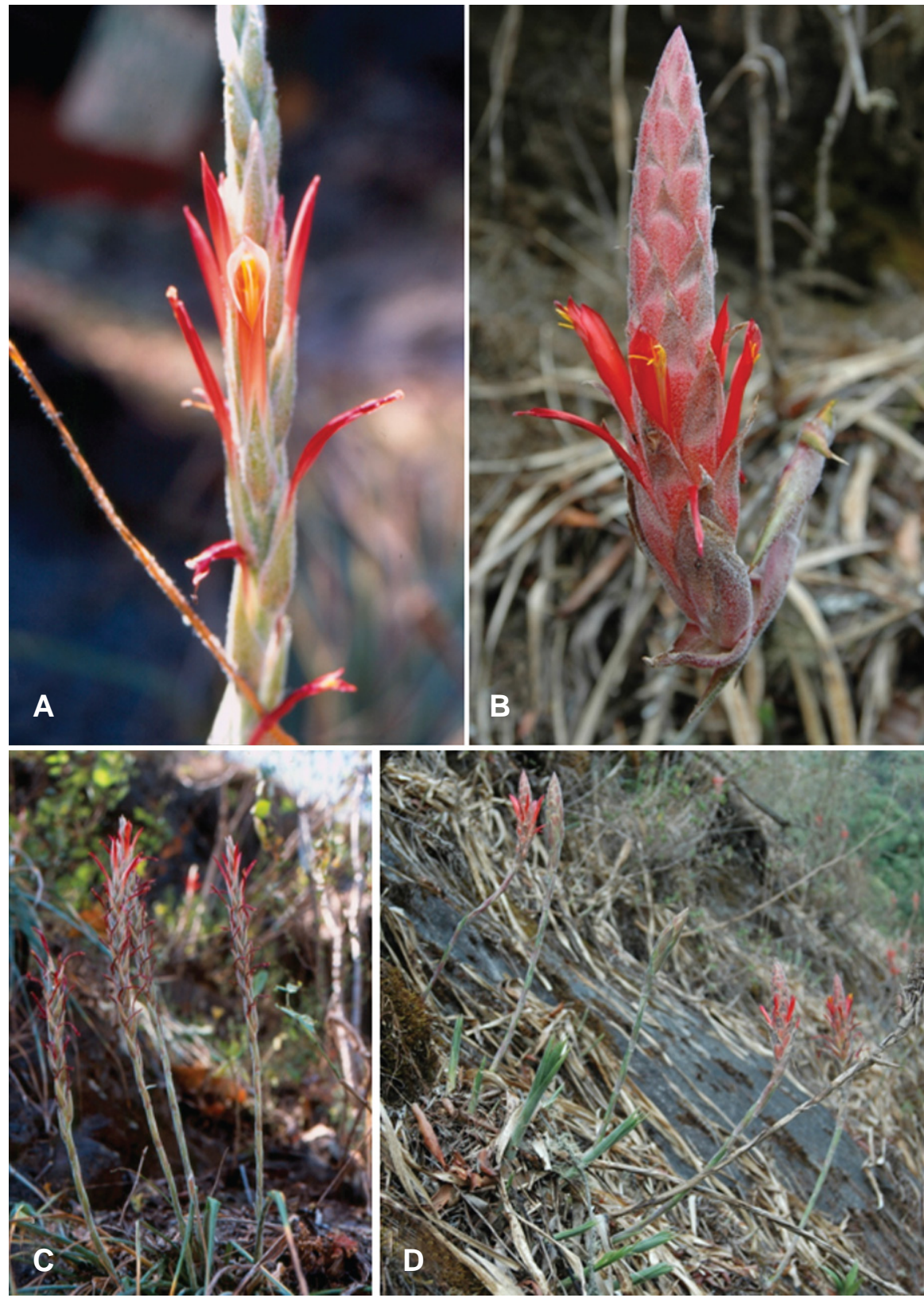

Fig. 1. A y C. Pitcairnia roseana L.B. Sm. (A. R. López-Ferrari et al. 2404, fotos A. Espejo). B y D. P. yocupitziae Espejo \& López-Ferrarri (Y. Ramírez-Amezcua et al. 969, fotos V. Steinmann). 
lepidotos; sépalos libres, verdes hacia la base, rojizos hacia el ápice, angostamente triangulares a lanceolados, acuminados, de $3.6 \mathrm{~cm}$ de largo, de 4.5 a $5 \mathrm{~mm}$ de ancho, densa y conspicuamente blanco-lepidotos, los dos posteriores carinados; pétalos libres, rojos, largamente espatulados, de 6.6 a $7 \mathrm{~cm}$ de largo, de 7 a 10 mm de ancho, agudos, sin apéndices basales; estambres subiguales, más cortos a ligeramente más largos que los pétalos, los filamentos blancos, filiformes, de 5.2 a $5.3 \mathrm{~cm}$ de largo; anteras amarillas, lineares, de 12 a $13 \mathrm{~mm}$ de largo; ovario casi totalmente súpero, verde, ovoide, de ca. $1 \mathrm{~cm}$ de largo, de 3.5 a $4 \mathrm{~mm}$ de diámetro; estilo blanco hacia la base, rojo en el ápice, linear, de $6.6 \mathrm{~cm}$ de largo; estigma rojo, del tipo conduplicado espiral (tipo II sensu Brown \& Gilmartin, 1984); cápsulas pardas claras, ovoides, de 9 a $12 \mathrm{~mm}$ de largo, de 5 a $7 \mathrm{~mm}$ de diámetro; semillas rojizas, fusiformes, de $1.5 \mathrm{~mm}$ de largo, bicaudadas, las caudas blancas, de ca. 1 mm de largo.

Tipo: Guerrero, General Heliodoro Castillo, $1.5 \mathrm{~km}$ al noroeste de Puerto

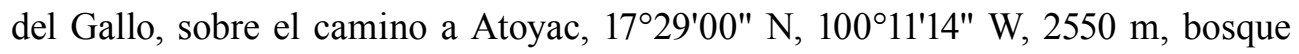
de pino encino, hierba perenne rupícola, flores rojas, 13.III.2007, Y. Ramírez-Amezcua, V. W. Steinmann y J. M. Porter 969 (Holotipo: UAMIZ; isotipos: ARIZ, IEB, MEXU).

Paratipo: Guerrero, General Heliodoro Castillo, $1.5 \mathrm{~km}$ al noroeste de

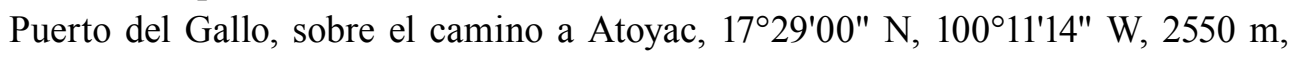
bosque de pino encino, hierba perenne rupícola, con frutos, 14.XII.2009, Y. Ramírez-Amezcua y V. W. Steinmann 1814 (IEB, UAMIZ).

Debido a sus inflorescencias y a sus brácteas florales densamente blanco-lepidotas, Pitcairnia yocupitziae es fenéticamente parecida a $P$. roseana L. B. Sm., planta de la cual difiere claramente en la forma y dimensiones de la inflorescencia y de las brácteas florales, además del tamaño de las flores (Fig. 1, Cuadro 1). Asimismo, las especies tienen preferencias ecológicas distintas, $P$. roseana habita en bosques tropicales caducifolios y encinares secos en un intervalo altitudinal que va de 350 a 2000 m s.n.m., en tanto que P. yocupitziae crece en bosques húmedos de pino-encino por arriba de los 2500 metros.

Pitcairnia yocupitziae se conoce hasta ahora únicamente de la localidad tipo en el estado de Guerrero, en el municipio de General Heliodoro Castillo, en donde crece en taludes rocosos, en bosques de pino-encino, en altitudes cercanas a los 2500 m s.n.m. (Fig. 2). 
Cuadro 1. Diferencias entre Pitcairnia yocupitziae Espejo et López-Ferrarri sp. nov. y P. roseana L. B. Sm.

\begin{tabular}{|c|c|c|}
\hline & Pitcairnia yocupitziae & Pitcairnia roseana \\
\hline $\begin{array}{l}\text { Inflorescencia excluyendo } \\
\text { al pedúnculo }\end{array}$ & $\begin{array}{l}\text { elipsoide a fusiforme, } 17-20 \\
\mathrm{~cm} \text { largo, } 4-5 \mathrm{~cm} \text { diámetro }\end{array}$ & $\begin{array}{l}\text { angostamente cilíndrica, 20-22 } \\
\mathrm{cm} \text { largo, } 1.5-2 \mathrm{~cm} \text { diámetro }\end{array}$ \\
\hline Brácteas florales & $\begin{array}{l}\text { largamente ovadas, rojas con } \\
\text { el ápice pardo, } 4.5-5.5 \mathrm{~cm} \\
\text { largo, } 15-17 \mathrm{~mm} \text { ancho }\end{array}$ & $\begin{array}{l}\text { ovadas a triangulares, verdes, } \\
1.5-2.3 \mathrm{~cm} \text { largo, } 7-8 \mathrm{~mm} \\
\text { ancho }\end{array}$ \\
\hline Sépalos & $\begin{array}{l}\text { angostamente triangulares a } \\
\text { lanceolados, } 3.6 \mathrm{~cm} \text { largo, } \\
4.5-5 \mathrm{~mm} \text { ancho }\end{array}$ & $\begin{array}{l}\text { angostamente elípticos, 2-2.2 } \\
\mathrm{cm} \text { largo, 5-6 mm ancho }\end{array}$ \\
\hline Pétalos & $\begin{array}{l}\text { largamente espatulados, 6.6-7 } \\
\mathrm{cm} \text { largo, 7-10 mm ancho }\end{array}$ & $\begin{array}{l}\text { espatulados a oblongo- } \\
\text { espatulados, } 4.7-5 \mathrm{~cm} \text { largo, } \\
6-7 \mathrm{~mm} \text { ancho }\end{array}$ \\
\hline Filamentos & 5.2-5.3 cm largo & $3.6-3.7 \mathrm{~cm}$ largo \\
\hline Anteras & 12-13 mm largo & 7-8 mm largo \\
\hline Estilo & ca. $6.6 \mathrm{~cm}$ largo & ca. 4 cm largo \\
\hline Hábitat & bosque de pino-encino & $\begin{array}{l}\text { bosque tropical caducifolio y } \\
\text { encinares secos }\end{array}$ \\
\hline Altitud & $2550 \mathrm{~m}$ & $350-2000 \mathrm{~m}$ \\
\hline Distribución en Mexico & Guerrero & $\begin{array}{l}\text { Durango, Guerrero, Jalisco, } \\
\text { Michoacán, Morelos, Nayarit, } \\
\text { Puebla }\end{array}$ \\
\hline
\end{tabular}

El epíteto específico hace referencia a Yocupitzia Ramírez Amezcua, colaboradora del Centro Regional del Bajío del Instituto de Ecología, quien colectó por primera vez plantas de esta notable especie.

La única población conocida hasta ahora de Pitcairnia yocupitziae forma una extensa aunque muy localizada colonia. Aparentemente, las plantas no son usadas por los habitantes de la región, por lo cual pensamos que $P$. yocupitziae no está sujeta a presiones antrópicas inmediatas; sin embargo y debido a la carencia de información detallada de la distribución precisa de la especie sugerimos su inclusión en la categoría Data Deficient (DD) de la UICN (Anónimo, 2003).

De los 18 géneros de Bromeliaceae presentes en México (Espejo-Serna et al., 2004), Pitcairnia es el tercero en número de taxa en el país, con 45 especies registradas (Espejo-Serna et al., 2004). Además, el género es notable por su alto nivel de endemis- 


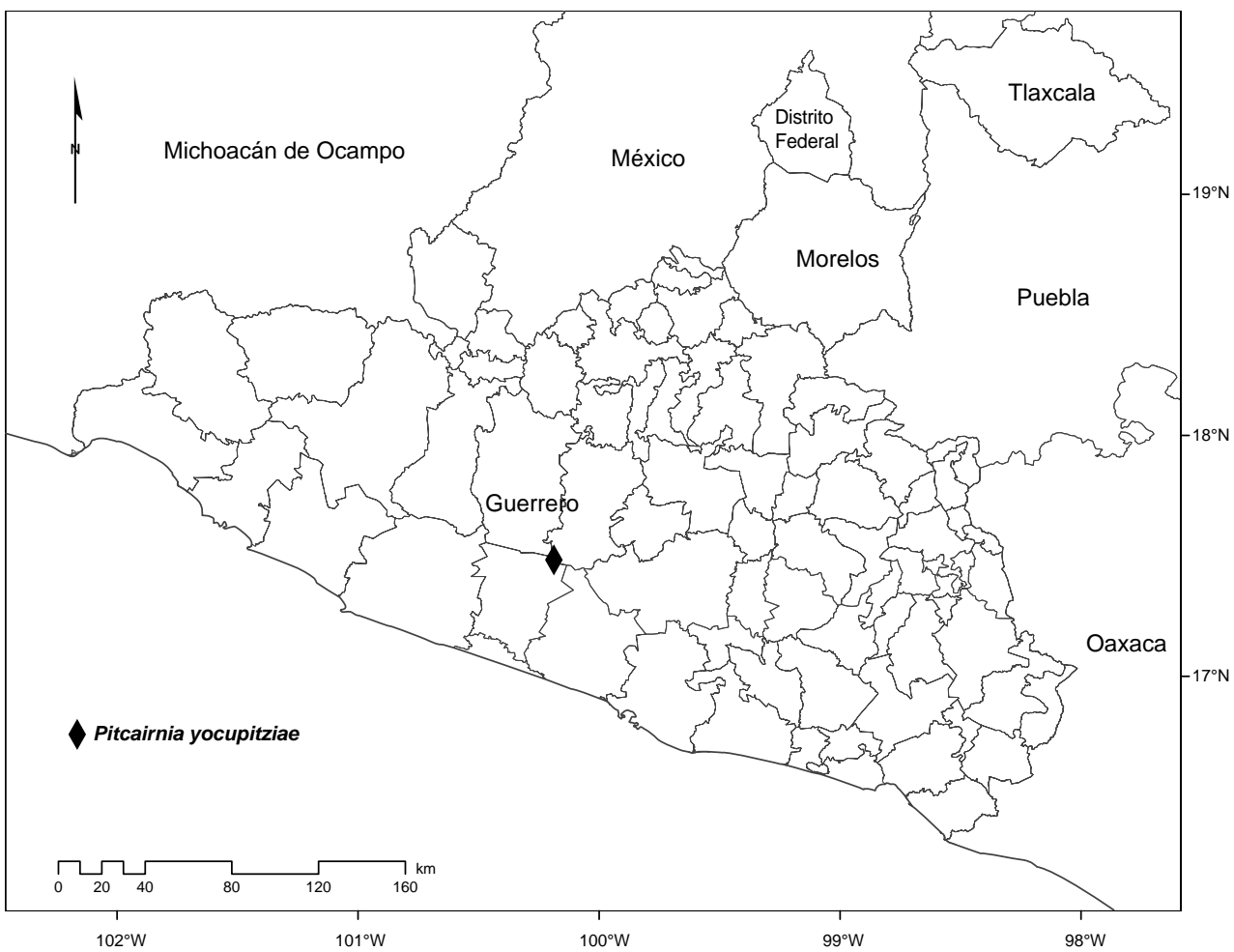

Fig. 2. Distribución conocida de Pitcairnia yocupitziae Espejo \& López-Ferrarri.

mo que alcanza 82.2\%. Del estado de Guerrero teníamos hasta ahora reportadas 14 especies (Pulido-Esparza et al., 2004) y con este nuevo hallazgo, dicho número se incrementa a 15. A continuación incluimos una clave de identificación para las mismas.

1 Hojas persistentes, con frecuencia pecioladas.

2 Brácteas florales de más de $2 \mathrm{~cm}$ de largo, imbricadas, más largas que los sépalos; hojas conspicuamente pecioladas

P. imbricata

2 Brácteas florales de menos de $1 \mathrm{~cm}$ de largo, no imbricadas, más cortas que los sépalos y que los pedicelos; hojas angostándose hacia la base pero no conspicuamente pecioladas.

3 Plantas de menos de $50 \mathrm{~cm}$ de alto; láminas de las hojas de 2 a $2.7 \mathrm{~cm}$ de ancho; brácteas florales de $5 \mathrm{~mm}$ de largo; pétalos rojos, de ca. $7 \mathrm{~cm}$ de largo P. oaxacana 
3 Plantas de 60 a $70 \mathrm{~cm}$ de alto; láminas de las hojas de (3-)4 a $5 \mathrm{~cm}$ de largo; brácteas florales de 7 a $9 \mathrm{~mm}$ de largo; pétalos amarillos, de 4 a $5 \mathrm{~cm}$ de largo P. flavescentia

1 Hojas deciduas a lo largo de una línea transversal, generalmente sésiles.

4 Escapo de la inflorescencia muy corto o ausente; pétalos provistos de una escama basal interna P. heterophylla

4 Escapo de la inflorescencia conspicuo; pétalos desprovistos de una escama basal interna.

5 Pedicelos de 2 a $4 \mathrm{~mm}$ de largo.

6 Brácteas florales glabras a glabrescentes P. cylindrostachya

6 Brácteas florales densa y persistentemente blanco-lepidotas.

7 Inflorescencia angostamente cilíndrica, de 1.5 a $2 \mathrm{~cm}$ de diámetro; brácteas florales verdes, de 1.5 a $2.3 \mathrm{~cm}$ de largo; pétalos de 4.7 a 5 $\mathrm{cm}$ de largo; filamentos de 3.6 a $3.7 \mathrm{~cm}$ de largo P. roseana

7 Inflorescencia elipsoide a fusiforme, de 4 a $5 \mathrm{~cm}$ de diámetro; brácteas florales rojas con el ápice pardo, de 4.5 a $5.5 \mathrm{~cm}$ de largo; pétalos de 6.6 a $7 \mathrm{~cm}$ de largo; filamentos de 5.2 a $5.3 \mathrm{~cm}$ de largo .. P. yocupitziae

5 Pedicelos de 4 a $20 \mathrm{~mm}$ de largo.

8 Pétalos de 7 a $10 \mathrm{~cm}$ de largo; sépalos de 3 a $3.5 \mathrm{~cm}$ de largo.

9 Cálices densamente tomentosos, aterciopelados, el tomento pardo oscuro a ferrugíneo P. modesta

9 Cálices glabros a glabrescentes, el indumento, cuando presente, blanquecino a pardo claro.

10 Sépalos esparcidamente lepidotos, las escamas estrelladoblanquecinas a pardo claras P. leprosa

10 Sépalos glabros P. hintoniana

8 Pétalos de 4 a $7 \mathrm{~cm}$ de largo; sépalos de 1.5 a $2.4 \mathrm{~cm}$ de largo.

11 Brácteas florales más cortas que los pedicelos; flores de $7 \mathrm{~cm}$ de largo P. mooreana

11 Brácteas florales del mismo tamaño o más largas que los pedicelos; flores de 4 a $5 \mathrm{~cm}$ de largo.

12 Hojas monomórficas; inflorescencia con 3 a 5 flores laxamente dispuestas P. tillandsioides

12 Hojas dimórficas; inflorescencia con más de 10 flores más o menos apretadamente dispuestas. 
13 Inflorescencia secundiflora; pedicelos de 10 a $14 \mathrm{~mm}$ de largo, aplanados P. pteropoda

13 Inflorescencia polística; pedicelos de 5 a $9 \mathrm{~mm}$ de largo, rollizos.

14 Inflorescencia tomentosa, de aspecto sucio o polvoriento; sépalos posteriores no alados P. sordida

14 Inflorescenciaglabra;sépalosposterioresconspicuamente alados P. karwinskyana

\section{AGRADECIMIENTOS}

Queremos agradecer a Victor W. Steinmann y a Yocupitzia Ramírez Amezcua por proporcionarnos el material de la nueva especie. Los curadores de los siguientes herbarios nos brindaron todas las facilidades para consultar sus colecciones CHAP, FCME, GH, ENCB, IBUG, IEB, MEXU, MICH, UAMIZ y US. El mapa fue realizado por Nancy Martínez-Correa y las fotografías de la nueva especie son obra de Victor W. Steinmann. Este trabajo se realizó gracias al apoyo del convenio 108215 del Consejo Nacional de Ciencia y Tecnología (CONACyT).

\section{LITERATURA CITADA}

Anónimo. 2003. Directrices para emplear los criterios de la Lista Roja de la UICN a nivel regional: Versión 3.0. Comisión de Supervivencia de Especies de la UICN. UICN, Gland, Suiza y Cambridge, Reino Unido. ii +26 pp.

Brown, G. K. y A. J. Gilmartin. 1984. Stigma structure and variation in Bromeliaceae neglected taxonomic characters. Brittonia 36: 364-374.

Espejo-Serna, A., A. R. López-Ferrari, I. Ramírez-Morillo, B. K. Holst, H. E. Luther y W. Till. 2004. Checklist of Mexican Bromeliaceae with notes on species distribution and levels of endemism. Selbyana 25: 33-86.

Pulido-Esparza, V. A., A. R. López-Ferrari y A. Espejo-Serna. 2004. Flora Bromeliológica del Estado de Guerrero: riqueza y distribución. Bol. Soc. Bot. Méx. 75: 55-104. 